\title{
Research on Enterprise Management Evaluation Model based on AHP Method
}

\author{
Hu Jixi \\ Pingxiang University, Pingxiang, Jiangxi, 337055, China
}

\begin{abstract}
Keywords: Analytic hierarchy process( AHP) ; enterprises management quality; evaluation index; weighted
\end{abstract} vector

\begin{abstract}
An effective evaluation of enterprises management quality is vital for improving the level of management. By analyzing the main factors related to enterprises management, we present a four level enterprises management quality system. We introduce an assessment model of enterprises management quality based on AHP. It is an effective way to guarantee the managing quality of enterprises.
\end{abstract}

\section{Introduction}

The quality of enterprise management is the main embodiment of enterprise intrinsic value. Effective evaluation of enterprise management value is the important condition for enterprises to carry out dynamic management and realize scientific management, and also an important reference for investors to choose the investment direction[1]. Therefore, the establishment of scientific and reasonable enterprise management quality evaluation model has important theoretical significance and practical application value[2,3]. Enterprise management quality assessment is often faced with a complex system of interrelated and mutually restrictive factors[4,5]. The evaluation process emphasizes the overall and systematic view of the problem, taking into account the role of the various aspects of the enterprise management system, in the fullest possible understanding of the company's intrinsic value on the basis of objective and fair to quantify the company's management value[6,7].

Analytic Hierarchy Process (AHP) is a comprehensive evaluation method for solving complex decision problems with multi-objective and multi-criteria. The method was proposed by Saaty et al. in the 1970s and introduced to China in the 1980s[8]. Because of its qualitative and quantitative combination of the characteristics of the decision-making factors, analytic hierarchy process quickly in all walks of life has been widely used. The basic idea is to decompose the complex decision problem into a multi-level structure model formed by factors with interrelated or affiliation, and to calculate the weight coefficient of each factor in the same layer by establishing the judgment matrix layer by layer[9,10]. Finally, The bottom layer is the overall weight of the target layer. On the detailed description of the hierarchy method and its application can refer to [11], the following use of analytic hierarchy process to establish the assessment model of enterprise management quality.

\section{Enterprise Management Evaluation Model}

\section{The establishment of hierarchical model}

To establish the hierarchical model of enterprise management quality, we must first determine the scientific and reasonable evaluation index system. This paper summarizes the existing research results, using the secondary index system, from the institutional mechanisms, management methods and corporate culture three dimensions (standard layer) to examine the quality of enterprise management. Each dimension is divided into four specific sub-elements (assessment indicators), together with the enterprise (program layer) to be evaluated, to form a four-level hierarchy model (Figure 1). Among them, the program layer includes three reference enterprises and a business to be evaluated, the three reference companies $D_{1}, D_{2}, D_{3}$, respectively, on behalf of the quality of management of quality enterprises, general enterprises and backward enterprises. This paper attempts to take three representative companies as a reference, given the level of management to be assessed. 
Figure 1shows that for each level of each factor on the target layer of the composition of the weight coefficient.

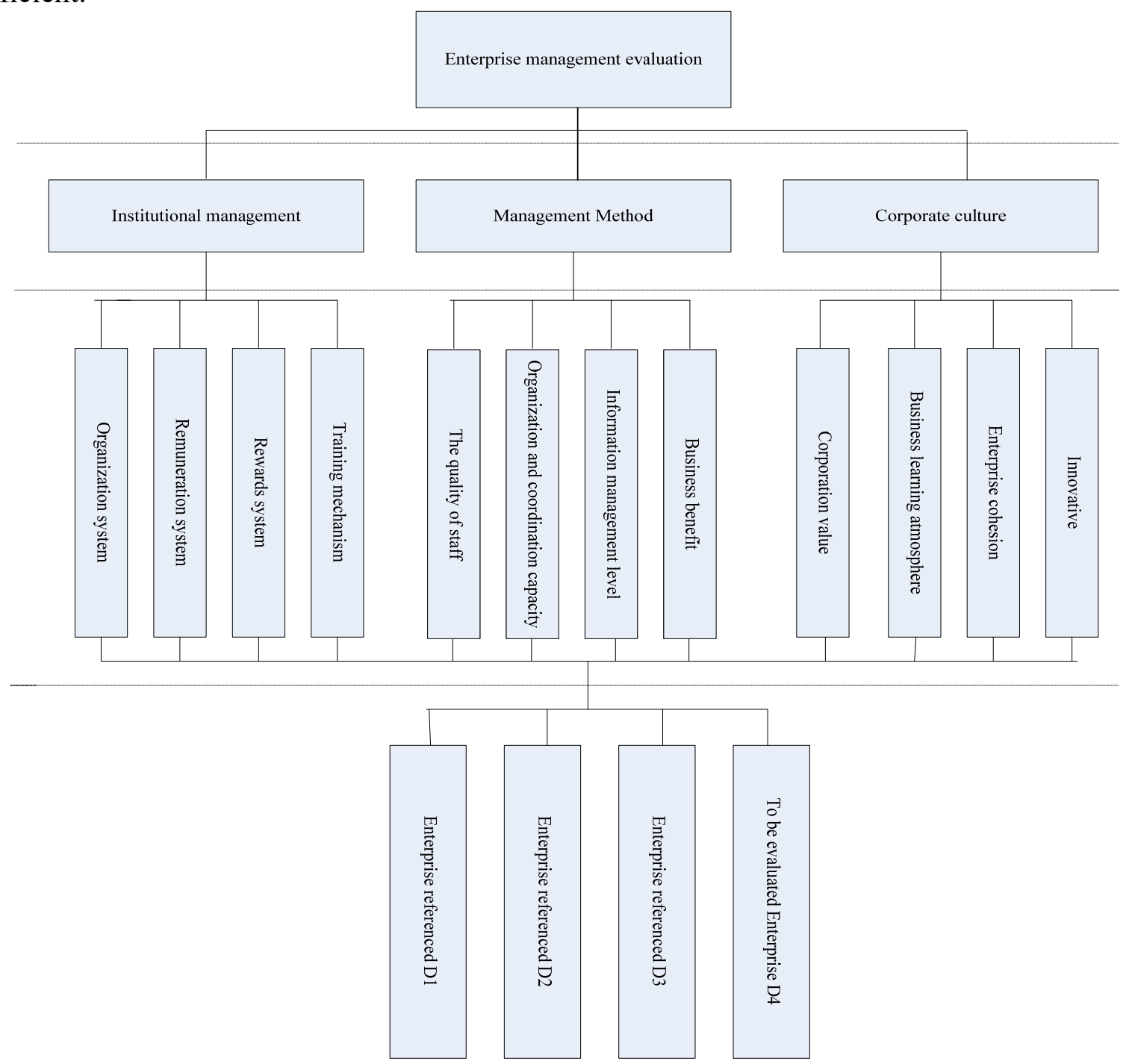

Figure 1. Hierarchical model of enterprise management quality evaluation

\section{Calculation of combination vector of index system}

In order to determine the combined weighting factor for each layer, we first establish the weighting coefficients for each element of each layer for each element of the upper layer. These weights are constructed by constructing a judgment matrix.

Suppose you want to calculate the influence of a certain layer's $n$ factors $P_{1}, P_{2}, \cdots, P_{n}$ on a factor $Q$ on the upper layer, and can be realized by constructing a judgment matrix. Let $A=\left(a_{i j}\right)_{n \times n}$ be a judgment matrix whose element $a_{i j}$ represents the ratio of and $P_{i}, P_{j}$ to the influence of $Q$ and satisfy the relation $a_{i j}=1 / a_{j i}$, then the eigenvector corresponding to the largest eigenvalue of matrix $A$ which denotes the influence weights of $P_{1}, P_{2}, \cdots, P_{n}$ with respect to $Q$. The value of the element $a_{i j}$ is generally given by Saaty's 1 - 9 ratio scale(Table 1$)$. 
Table 1. Discription of different $a_{i j}$

\begin{tabular}{|c|l|}
\hline$a_{i j}$ & Discription \\
\hline 1 & $\mathrm{P}_{\mathrm{i}}$ is as important as $\mathrm{P}_{\mathrm{j}}$ \\
\hline 3 & $\mathrm{P}_{\mathrm{i}}$ is a little importanter than $\mathrm{P}_{\mathrm{j}}$ \\
\hline 5 & $\mathrm{P}_{\mathrm{i}}$ is importanter than $\mathrm{P}_{\mathrm{j}}$ \\
\hline 7 & $\mathrm{P}_{\mathrm{i}}$ is much importanter than $\mathrm{P}_{\mathrm{j}}$ \\
\hline 9 & $\mathrm{P}_{\mathrm{i}}$ is really much importanter than $\mathrm{P}_{\mathrm{j}}$ \\
\hline $2,4,6,8$ & The ratio of $\mathrm{P}_{\mathrm{i}}$ to $\mathrm{P}_{\mathrm{j}}$ is between the two adjacent levels \\
\hline $1 / 2,1 / 3, \ldots, 1 / 9$ & The ratio of $\mathrm{P}_{\mathrm{j}}$ to $\mathrm{P}_{\mathrm{i}}$ is the reciprocal of the value of $a_{i j}$ above \\
\hline
\end{tabular}

The coherence ratio $C R=\frac{C I}{R I}$ can be used to test the consistency of the judgment matrix, where $C I=\frac{\lambda-n}{n-1}, \lambda$ and $\mathrm{n}$ represent the maximum eigenvalue and order of the judgment matrix, respectively, and RI is the random consistency index ( Table 2). In general, when $C R<0.1$, it can be considered that the judgment matrix passes the test and has satisfactory consistency.

Table 2. Random Consistency Index RI Values

\begin{tabular}{|l|l|l|l|l|l|l|l|l|l|l|l|}
\hline $\mathrm{n}$ & 1 & 2 & 3 & 4 & 5 & 6 & 7 & 8 & 9 & 10 & 11 \\
\hline $\mathrm{RI}$ & 0 & 0 & 0.58 & 0.90 & 1.12 & 1.24 & 1.32 & 1.41 & 1.45 & 1.49 & 1.51 \\
\hline
\end{tabular}

The weighting coefficients for the upper-level factors of each layer factor in Fig. 1 are calculated by constructing a judgment matrix. All elements of the judgment matrix are obtained by consulting expert professors in business management.

The judgment matrix of the first-level indicator about the target layer is $A=\left(\begin{array}{ccc}1 & 1 / 4 & 1 / 3 \\ 4 & 1 & 2 \\ 3 & 1 / 2 & 1\end{array}\right)$, Where the maximum eigenvalue of matrix $A$ is $\lambda=3.0183$, the corresponding eigenvector is $w^{(2)}=\left[\begin{array}{lll}0.1220 & 0.5584 & 0.3196\end{array}\right]$. The coherence ratio is $C R^{(2)}=0.0158<0.1$, so pass the consistency test.

We use the same method to construct the judgment matrix of the secondary index on each factor (institutional mechanism, management method and corporate culture) of the primary index, respectively $B_{1}=\left(\begin{array}{cccc}1 & 1 / 3 & 1 / 5 & 1 / 3 \\ 3 & 1 & 1 / 2 & 1 \\ 5 & 2 & 1 & 2 \\ 3 & 1 & 1 / 2 & 1\end{array}\right), \quad B_{2}=\left(\begin{array}{cccc}1 & 2 & 1 & 3 \\ 1 / 2 & 1 & 1 / 2 & 1 \\ 1 & 2 & 1 & 2 \\ 1 / 3 & 1 & 1 / 2 & 1\end{array}\right) B_{3}=\left(\begin{array}{cccc}1 & 1 / 3 & 1 / 2 & 1 / 4 \\ 3 & 1 & 2 & 1 \\ 2 & 1 / 2 & 1 & 2 \\ 4 & 1 & 1 / 2 & 1\end{array}\right)$.

The maximum eigenvalues of the three matrices are $\lambda_{1}=4.0042, \lambda_{2}=4.0104, \lambda_{3}=4.1421$, respectively. The corresponding eigenvectors are $\omega^{(31)}=\left[\begin{array}{lllll}0.0819 & 0.2346 & 0.4488 & 0.2346\end{array}\right]$, $\omega^{(32)}=\left[\begin{array}{lllll}0.3509 & 0.1891 & 0.3509 & 0.1091\end{array}\right], \quad \omega^{(33)}=\left[\begin{array}{lllll}0.0934 & 0.2536 & 0.1766 & 0.4763\end{array}\right]$. The correlation ratios were $C R^{(31)}=0.0016, C R^{(32)}=0.0039, C R^{(33)}=0.045$ and the three judgment matrices passed the consistency test.

Consider the weight vector of each factor in the program layer for the secondary indicator. For the sake of simplicity, this paper only considers that the three reference firms $D_{1}, D_{2}$, and $D_{3}$ have the same importance for the four secondary indicators belonging to the same dimension. All the factors of the whole program layer correspond to the first four factors of the secondary index. 
The judgment matrix for the first four factors is:

$$
C_{k}=\left(\begin{array}{cccc}
1 & 2 & 3 & c_{14}^{(k)} \\
1 / 2 & 1 & 2 & c_{24}^{(k)} \\
1 / 3 & 1 / 2 & 1 & c_{34}^{(k)} \\
1 / c_{14}^{(k)} & 1 / c_{24}^{(k)} & 1 / c_{34}^{(k)} & 1
\end{array}\right), k=1,2,3,4
$$

The judgment matrix for the middle four factors is:

$$
C_{k}=\left(\begin{array}{cccc}
1 & 3 & 5 & c_{14}^{(k)} \\
1 / 3 & 1 & 3 & c_{24}^{(k)} \\
1 / 5 & 1 / 3 & 1 & c_{34}^{(k)} \\
1 / c_{14}^{(k)} & 1 / c_{24}^{(k)} & 1 / c_{34}^{(k)} & 1
\end{array}\right), k=5,6,7,8
$$

While the judgment matrix for the last four factors is:

$$
C_{k}=\left(\begin{array}{cccc}
1 & 3 & 6 & c_{14}^{(k)} \\
1 / 3 & 1 & 3 & c_{24}^{(k)} \\
1 / 6 & 1 / 3 & 1 & c_{34}^{(k)} \\
1 / c_{14}^{(k)} & 1 / c_{24}^{(k)} & 1 / c_{34}^{(k)} & 1
\end{array}\right), k=9,10,11,12
$$

Where $C_{i 4}^{k}, i=1,2,3$ represents the ratio of $D_{i}$ and $D_{4}$ to the effect of the upper kth factor, and the value varies with the change of the firm to be evaluated. Once the values of $C_{i 4}^{k}, i=1,2,3, k=1,2, \cdots, 12$ are obtained, the weights of each factor of each firm on the upper layer of the scheme layer can be obtained, The composition of the layer.

\section{Empirical Research}

It is known that the value of $C_{i 4}^{k}$ corresponding to the enterprise to be determined is shown in Table 3. The weight vector of each factor can be obtained by calculating the eigenvector of each judgment matrix. $w_{k}^{(4)}$ and the consistency ratio $C R_{k}^{(4)}$, the relevant calculation results are shown in Table 4. From the value of $C R_{k}^{(4)}$, it can be seen that the judgment matrix involved passes the consistency test.

Table 3. The value of $C_{14}^{k}, C_{24}^{k}, C_{34}^{k}$

\begin{tabular}{|c|c|c|c|c|c|c|c|c|c|c|c|c|}
\hline $\mathrm{k}$ & 1 & 2 & 3 & 4 & 5 & 6 & 7 & 8 & 9 & 10 & 11 & 12 \\
\hline$C_{14}^{k}$ & 2 & 3 & 4 & 1 & 4 & 5 & 3 & 1 & 5 & 2 & 1 & 3 \\
\hline$C_{24}^{k}$ & 1 & 2 & 3 & $1 / 3$ & 2 & 3 & 1 & $1 / 3$ & 2 & 1 & $1 / 3$ & 2 \\
\hline$C_{34}^{k}$ & $1 / 2$ & 1 & 2 & $1 / 5$ & 1 & 2 & $1 / 4$ & $1 / 5$ & 1 & $1 / 2$ & $1 / 6$ & $1 / 5$ \\
\hline
\end{tabular}


Table 4. Part of the calculation results regrading Solution layer D

\begin{tabular}{|c|c|c|c|c|c|c|}
\hline $\mathrm{k}$ & 1 & 2 & 3 & 4 & 5 & 6 \\
\hline \multirow{3}{*}{$\mathrm{w}^{(3)}$} & 0.01 & 0.03 & 0.05 & 0.03 & 0.20 & 0.11 \\
\hline \multirow{3}{*}{$w_{k}^{(4)}$} & 0.42 & 0.46 & 0.47 & 0.33 & 0.55 & 0.56 \\
\cline { 2 - 7 } & 0.23 & 0.26 & 0.28 & 0.16 & 0.24 & 0.25 \\
\cline { 2 - 7 } & 0.12 & 0.14 & 0.16 & 0.09 & 0.10 & 0.11 \\
\cline { 2 - 7 } & 0.23 & 0.14 & 0.10 & 0.42 & 0.12 & 0.08 \\
\hline$C_{k}^{(4)}$ & 0.00 & 0.00 & 0.01 & 0.01 & 0.02 & 0.03 \\
\hline$C R_{k}^{(4)}$ & 0.00 & 0.00 & 0.01 & 0.01 & 0.02 & 0.04 \\
\hline $\mathrm{k}$ & 7 & 8 & 9 & 10 & 11 & 12 \\
\hline \multirow{2}{*}{$\mathrm{w}^{(3)}$} & 0.20 & 0.06 & 0.03 & 0.08 & 0.06 & 0.15 \\
\hline \multirow{3}{*}{$w_{k}^{(4)}$} & 0.52 & 0.39 & 0.58 & 0.50 & 0.40 & 0.52 \\
\cline { 2 - 7 } & 0.20 & 0.15 & 0.22 & 0.20 & 0.15 & 0.23 \\
\cline { 2 - 7 } & 0.08 & 0.07 & 0.09 & 0.07 & 0.06 & 0.06 \\
\cline { 2 - 7 } & 0.20 & 0.39 & 0.11 & 0.22 & 0.40 & 0.19 \\
\hline$C I_{k}^{(4)}$ & 0.01 & 0.01 & 0.01 & 0.01 & 0.01 & 0.06 \\
\hline$C R_{k}^{(4)}$ & 0.02 & 0.02 & 0.01 & 0.01 & 0.01 & 0.07 \\
\hline
\end{tabular}

We use the $w_{k}^{(4)}$ of the three-row and 12-column matrix $w^{(4)}$ in Table3, and then use the $w^{(4)}=w^{(4)} w^{(3)}$ to obtain the combination of the solution layer Vector, ie $w^{(4)}=\left[\begin{array}{llll}05031 & 0.2171 & 0.0895 & 0.1902\end{array}\right]$. The results show that the management quality of the enterprise is close to the average level. It is easy to verify that the above model can also be used as the evaluation value of the enterprise management value by combining the consistency test in accordance with.

\section{Summary}

In recent years, with the development of network and flipped classroom concept, short and pithy micro courses are more and more young learners of all ages. Based on the analysis of the concept and characteristics of the micro course, this paper puts forward the methods and achievements of introducing the corpus in the course design and development of oral English teaching in order to improve the effectiveness and pertinence of the micro course. This paper discusses the feasibility and application of the introduction of corpus research to the development of micro courses, and puts forward the model of the design and development of the corpus based micro course.

\section{References}

[1]. Ho W, Xu X, Dey P K. Multi-criteria decision making approaches for supplier evaluation and selection: A literature review[J]. European Journal of operational research, 2010, 202(1): 16-24.

[2]. Lee A H I, Chen W C, Chang C J. A fuzzy AHP and BSC approach for evaluating performance of IT department in the manufacturing industry in Taiwan[J]. Expert systems with applications, 2008, 34(1): 96-107.

[3]. Tzeng G H, Chiang C H, Li C W. Evaluating intertwined effects in e-learning programs: A novel hybrid MCDM model based on factor analysis and DEMATEL[J]. Expert systems with Applications, 2007, 32(4): 1028-1044.

[4]. Skibniewski M J, Chao L C. Evaluation of advanced construction technology with AHP method[J]. Journal of Construction Engineering and Management, 1992, 118(3): 577-593. 
[5]. Govindan K, Rajendran S, Sarkis J, et al. Multi criteria decision making approaches for green supplier evaluation and selection: a literature review[J]. Journal of Cleaner Production, 2015, 98: 66-83.

[6]. Lu R, Liu R, Hu X. Overview on Research of Vegetables Channels Performance[J]. Management \& Engineering, 2015 (19): 81.

[7]. Oztaysi B. A decision model for information technology selection using AHP integrated TOPSIS-Grey: The case of content management systems[J]. Knowledge-Based Systems, 2014, 70: 44-54.

[8]. Cai T, Dai H C, Song H X. Research on the evaluation model of brand competitiveness of power enterprises based on the fuzzy comprehensive evaluation method[J]. Fuzzy System and Data Mining: Proceedings of FSDM, 2016, 281: 17.

[9]. Hao F, Yuan Z, Gu Q, et al. Research on the load management and assessment system for the high energy-consuming enterprises based on key index data analysis[C]/Electricity Distribution (CICED), 2016 China International Conference on. IEEE, 2016: 1-6.

[10]. Cui L, Yuan N. Empirical Research on Enterprise Manager Evaluation Model Based on Meta-competency[J]. China Labor, 2015, 18: 016.

[11]. Xiong G, Chen Y, Shang X, et al. AHP fuzzy comprehensive method of supplier evaluation in social manufacturing mode[C]//Intelligent Control and Automation (WCICA), 2014 11th World Congress on. IEEE, 2014: 3594-3599.

[12]. Wu D, Olson D L, Dolgui A. Decision making in enterprise risk management: A review and introduction to special issue[J]. 2015. 